\title{
¿También un arte 'macro-levantino'? El arquero de grandes dimensiones de Val del Charco del Agua Amarga (Alcañiz, Teruel)
}

\author{
Is there also a 'macro-Levantine rock art'? The large archer of Val del Charco del Agua \\ Amarga (Alcañiz, Teruel)
}

\author{
Manuel Bea (*) \\ José Ignacio Royo (**)
}

\section{RESUMEN}

El análisis mediante tratamiento digital de la imagen de una serie de restos pictóricos en el abrigo de Val del Charco nos permite identificar la figura de un gran arquero levantino. Son excepcionales sus extraordinarias dimensiones (130 cm de altura estimada), así como su clasificación dentro del denominado Horizonte Centelles. Su ubicación central y elevada en el abrigo subraya su posición dominante acentuando la visibilidad del motivo también desde el entorno inmediato. El carácter singular de esta representación podría igualmente abundar en la consideración del abrigo como un centro de agregación. La definición de este motivo aparece como un caso único en el panorama del arte levantino que complementa las caracterizaciones aportadas para el horizonte estilístico Centelles sin invalidarlas en modo alguno.
Centelles stylistic horizon. The motive is located in the central-upper part of the decorated panel, something that highlights its dominant position, emphasizing the visibility of the figure in the surroundings. The singular aspect of this representation could also point out the aggregation character of the rock art shelter, already underlined in other studies. Therefore, this motive appears as a unique case in the Levantine rock art panorama. In any case, all these particulars do not invalid the distinctive definitions of the Centelles stylistic horizon, appearing as a complement to them.

Palabras clave: Val del Charco; Maestrazgo; Aragón; Península Ibérica; Arte levantino; Holoceno; Horizonte Centelles; Calcos digitales.

Key words: Val del Charco; Maestrazgo region; Aragón; Iberian Peninsula; Levantine rock art; Holocene; Centelles stylistic Horizon; Digital traces.

\section{INTRODUCCIÓN}

A raíz del descubrimiento y posterior estudio de la Cueva del Chopo (Obon, Teruel), donde se documentaron una serie de antropomorfos levantinos de más de un metro de altura, se ha venido especulando con la posibilidad de que algunas representaciones excedieran con mucho los cánones de tamaños medios del corpus iconográfico de los antropomorfos levantinos (Picazo et al. 20012002: 59 y n. 7). El presente trabajo da a conocer un arquero cuyas características, estilo y dimensiones permiten plantear, con ciertas garantías, la 
existencia de escasos pero cada vez más significativos antropomorfos que representan figuras con un marcado carácter singular. Además se localiza en Val del Charco del Agua Amarga, no de los conjuntos parietales levantinos más importantes de Aragón, no solo por la calidad y número de sus representaciones, sino también por su especial significación dentro de la ordenación territorial de las estaciones rupestres del Bajo Aragón y de la cuenca del río Guadalope (Fig. 1).

El abrigo decorado se encuentra en una zona muy transitada en la que se extienden, desde antiguo, campos de cultivo. Pese haber sido una vía de comunicación de primer orden, las pinturas de Val del Charco del Agua Amarga (Alcañiz, Teruel) no fueron descubiertas hasta 1913. Su importancia fue reconocida desde el inicio de los trabajos sobre el conjunto pero Cabré (1915) no las publicará hasta dos años más tarde. Su obra clásica,
Arte Rupestre en España, será de referencia para el conjunto rupestre hasta la exhaustiva síntesis de Almagro (1956) que incluye los calcos de Ripoll, y supera la breve publicación de Tomás Maigí (1951) referida, esencialmente, a los materiales recuperados en un sondeo arqueológico realizado en el abrigo. El siguiente estudio pormenorizado es la monografía de Beltrán (1970) que incorpora calcos y dibujos de Cabré y Almagro y se convertirá en el análisis más completo que se le dedica durante más de 30 años. Trabajos posteriores introducirán pequeñas correcciones, sobre todo en los calcos (Beltrán 1968). Abundan las citas al conjunto en numerosos artículos y estudios, entre los que destacamos una breve pero interesante síntesis del abrigo (Royo y Benavente 1999).

Con la pretensión de preservar el conjunto, entre 1999 y comienzos del 2000 se emprenden tareas de mantenimiento, conservación y docu-

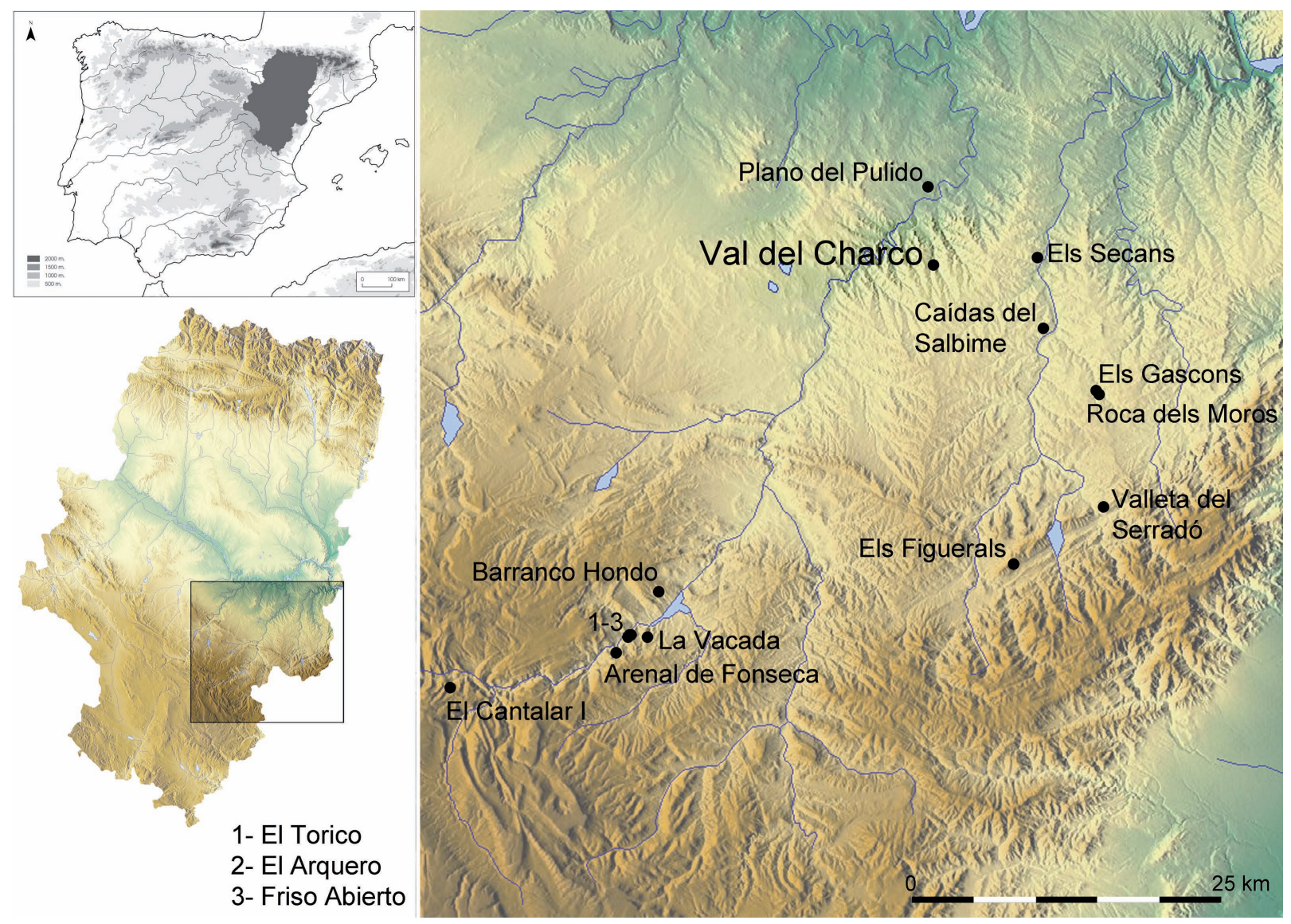

Fig. 1. Mapa de localización del abrigo de Val del Charco y conjuntos levantinos próximos. 
mentación. Como parte culminante de las mismas, se encargó a E. Guillamet un completo proceso de restauración y limpieza de los paneles pintados. $\mathrm{Su}$ conclusión dejó al descubierto la gran riqueza pictórica del abrigo que pasó de contar con 73 figuras a 109. A la vista de las novedades, se planteó un cuidadoso proyecto de estudio, dirigido por Beltrán, cuyo fruto fue una monografía de obligada referencia para el conocimiento tanto del abrigo como del arte levantino aragonés (Beltrán 2002). Una de las nuevas interpretaciones era un arquero de grandes dimensiones identificado por el propio E. Guillamet y J. I. Royo.

En 2011, los trabajos de revisión en Val del Charco documentaron profusamente restos de pigmento que ya se habían identificado como partes integrantes de la gran figura de arquero, si bien nunca se había completado el análisis en profundidad del motivo. Por cuestiones que tratamos más adelante, Beltrán (2000: 72, 2002: 138-139) no llegaría a aceptar esta representación como otros autores (Picazo et al. 2001-2002; Utrilla 2005: 363).

Los calcos se han obtenido mediante procedimiento digital, combinando esta metodología bien definida en trabajos previos (Domingo y López 2002; Martínez-Bea 2009) con el tratamiento digital de las imágenes mediante ImageJ. Este programa apareció como una herramienta de mejora de las imágenes multiespectrales empleadas en teledetección. A partir del mismo, se desarrolló DStretch, una aplicación para su uso con el citado software, especialmente diseñada para el análisis de conjuntos con arte rupestre (Harman 2005). Esta aplicación permite la observación mejorada de las imágenes rupestres al aumentar la saturación de los colores, mediante la transformación Karhunen-Loève (KLT), y analizar la matriz de covarianza de los colores representados en la fotografía. Gracias a la imagen de falso color resultante, con grandes variaciones en el contraste de los tonos, la visión del motivo o composición es más clara. Cada imagen se transforma de modo independiente, debido a su particular distribución de colores, a partir de las tonalidades del soporte rocoso y de los pigmentos empleados en la elaboración de los motivos rupestres.

La aplicación de esta herramienta se ha ido generalizando con el tiempo, obteniéndose interesantes resultados en conjuntos levantinos (Bea 2012) y esquemáticos (Díaz-Andreu 2012), que han documentado nuevas representaciones o detalles de otras ya conocidas.

\section{CASO DE ESTUDIO}

El arquero de Val del Charco del Agua Amarga (Alcañiz, Teruel) presenta un mal estado de conservación global debido, en gran medida, a su ubicación. El espacio decorado del abrigo cuenta con dos cavidades bien diferenciadas por una superficie convexa central que sirve de transición entre ambas. Esta zona debió estar profusamente decorada, ya que se documentaron representaciones pictóricas en todo su desarrollo vertical. También es la parte que más ha sufrido la circulación del agua y la presencia de algas o cianobacterias sobre todo en los brazos, cuerpo y cabeza del arquero. Estos elementos han determinado la desplacación de la superficie rocosa, así como el lavado de las pinturas con pérdida evidente de pigmento y de intensidad cromática. Esta circunstancia y las grandes dimensiones de la representación habrían impedido interpretarla en los términos en que lo hacemos ahora.

El motivo analizado está conformado por las figuras 64 (trazo alargado), 65 (línea quebrada), 66 (grupo de líneas formando un haz), 67 (grupo con dos líneas finas y paralelas), 68 y 69 de Beltrán (2002: 136-139). El motivo 68 se describe como una línea gruesa angular con "una inflexión o quiebro en su parte media central y en la terminación de su extremo izquierdo", que podría ser "el esquema del techo con dos vertientes de una choza" (Beltrán 1970: 79), una interpretación que se pone en duda en el estudio más reciente del conjunto (Beltrán 2002: 138). El motivo 69 se define como figura informe. Para tratar de reflejar con mayor exactitud la realidad de los motivos los describimos de nuevo (Fig. 2).

Motivo 64: restos lineales mal conservados. El mayor alcanza los $6,5 \mathrm{~cm}$ de longitud y $1 \mathrm{~cm}$ de grosor. Los consideramos parte del desarrollo de la pierna izquierda. Color: Munsell 7.5 R 5/6.

Motivo 65: extremo de arco. Trazo lineal curvo de desarrollo vertical en su primer tramo que adopta una disposición diagonal hacia la derecha en la zona superior. Mide $15 \mathrm{~cm}$ de longitud. Munsell 7.5 R 5/6.

Motivo 66: haz de flechas. Tres trazos lineales, paralelos entre sí y con un desarrollo ligeramente 

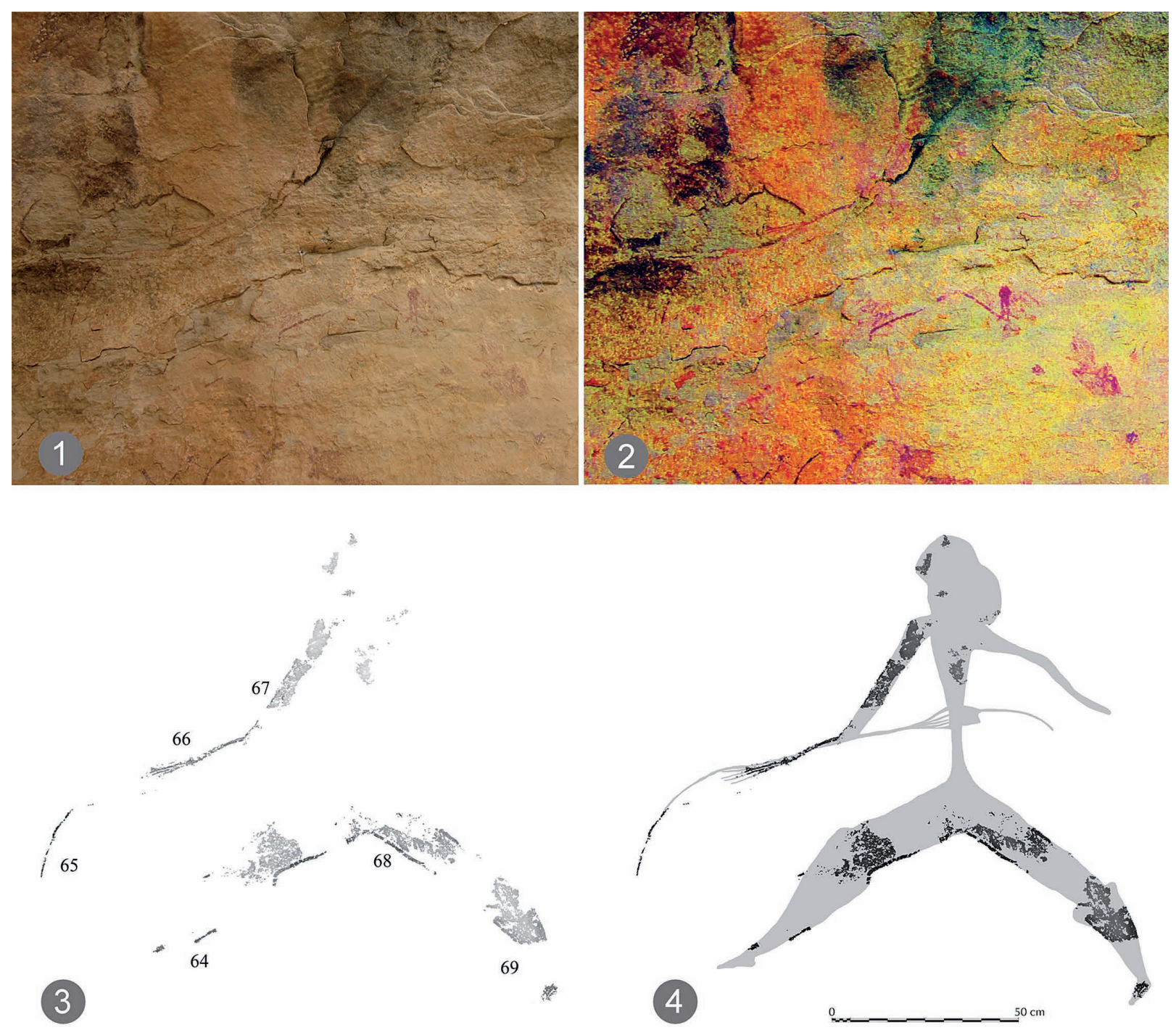

Fig. 2. 1. Fotografía original del motivo. 2. Fotografía del motivo tratada mediante DStretch. 3. Calco del motivo de arquero de grandes dimensiones de Val del Charco. 4. Propuesta de reconstrucción del motivo, basada en el motivo 21 del mismo abrigo.

diagonal que llegan a 24,6 cm de longitud máxima. Color: Munsell 7.5 R 4/4.

Motivo 67: brazo izquierdo. Se aprecian dos trazos lineales diagonales y paralelos entre sí bastante desvaídos. Con el tratamiento digital de la imagen hemos identificado un pigmento muy desvaído, prácticamente imperceptible, que aparece como parte del relleno de la extremidad. Color: Munsell 7.5 R 4/4.

Motivo 68: piernas. Está formado por una línea de $1,5 \mathrm{~cm}$ de grosor que se inicia en la parte iz- quierda con una inflexión de unos $55^{\circ}$ para seguir en diagonal ascendente hacia la derecha hasta los $23,3 \mathrm{~cm}$. Tras una nueva inflexión el trazo continúa en diagonal descendente otros $14 \mathrm{~cm}$. A 8 $\mathrm{cm}$ del trazo mencionado, en la zona derecha, y en paralelo, se advierte otro elemento lineal que delimitaría el contorno de la pierna derecha. Entre estos dos trazos se observan unas manchas difusas que se corresponderían con un relleno interior. Este mismo tipo de relleno se aprecia parcialmente en la zona izquierda del motivo. 
Beltrán (2002: 138) se refiere a la inflexión del extremo izquierdo del trazo como el ángulo inferior de la rodilla que aparecería flexionada en el sentido de la carrera. Sin embargo, consideramos que se trata en realidad de una marcada inflexión de la jarretera o musculatura del gemelo del arquero, exactamente igual a la observada en los motivos 3 y 21 del mismo abrigo. Esta interpretación permite dar sentido al trazo 64, que aparecería como el desarrollo del gemelo. Color: Munsell 7.5 R 5/6.

Motivo 69: pierna derecha. Restos de pigmento que marcan ligeramente el silueteado exterior de la misma naturaleza que el observado para el motivo anterior y el relleno interior algo más difuminado. Se correspondería con la pantorrilla del arquero, apreciándose el volumen muscular del gemelo. Interpretamos el resto informe a $11 \mathrm{~cm}$ por debajo como la zona del tobillo o parte del pie. Color: Munsell 7.5 R 5/6.

Solo con la aplicación DStretch hemos podido advertir una serie de restos pictóricos que, por su alto grado de degradación, apenas resultaban visibles a simple vista. Tal es el caso de parte del relleno interior del brazo más adelantado y de una serie de manchas sin gran definición que formarían parte del cuerpo y quizá de la cabeza.

A tenor de lo expuesto, los motivos referidos conformarían un figura de arquero orientada a la izquierda con piernas abiertas en ángulo de $125^{\circ}$, dentro de los parámetros apuntados para los arqueros tipo Centelles (Villaverde et al. 2006: 184), y cuyas proporciones alcanzarían, al menos, los $130 \mathrm{~cm}$ de altura. La interpretación conjunta de los motivos referidos como un arquero hace que sus dimensiones resulten extraordinarias. Seguramente por este motivo Beltrán llega a referirse a esta figura como conjetural. Describe las diferentes manchas y trazos de pigmento conservadas, identifica y caracteriza los principales elementos de dicho arquero pero, a continuación, niega su interpretación como tal (Beltrán 2002: 138).

Salvando las diferencias en cuanto a sus dimensiones, el motivo 21 del mismo abrigo parece guardar la mayor afinidad con él: arco y haz de flechas en disposición idéntica, grado de apertura de las piernas, volumen de los gemelos o las jarreteras bien marcadas. La línea de rotura que destaca muy marcadamente la mitad inferior de la pierna es un patrón muy característico. Observa- mos el mismo tratamiento en el motivo 3 de Val del Charco, así como en arqueros de Els Secans, La Vacada, Valleta del Serradó, El Garroso en Teruel o los de Mas del Ous, Centelles o algunas de las figuras desaparecidas dels Cavalls en Castellón (Fig. 3).

\section{CONCLUSIONES}

Atendiendo a las características apuntadas y, sobre todo, a la similitud estilística que guarda la figura que presentamos con el motivo 21 de Val del Charco, consideramos factible, a priori, clasificarlo en la categoría de arquetipo robusto $\mathrm{u}$ horizonte Centelles. Las grandes proporciones del arquero hacen que nos planteemos algunas cuestiones relativas al horizonte donde lo englobamos. La definición estilística referida ha sido tratada en diferentes contextos espaciales (Domingo 2005 y 2006; Martínez-Bea 2005) y estudios concretos (Villaverde et al. 2006). Sin embargo las dimensiones del sujeto de nuestro análisis rebasan con mucho la media de los arqueros de tipo Centelles y el tamaño de figuras, ya grandes dentro del horizonte reseñado, como el arquero de Cova Remigia $\mathrm{V}$ con casi $50 \mathrm{~cm}$; los restos de unas piernas de antropomorfo en Tolls Alts (Villaverde et al. 2006: 190, fig. 9) y otros ejemplos de la zona central del País Valenciano, como la mitad inferior de una "figura humana de cintura estrechísima y piernas gruesas" de la cavidad II de Cueva de la Araña (Hernández-Pacheco 1924: 99-100), para la que se ha estimado una altura de $80 \mathrm{~cm}$, así como grandes representaciones humanas del Cinto de las Letras. Pensamos, en especial, en los motivos 1a y 2c, interpretados como las pantorrillas $\mathrm{y}$ pies de dos figuras humanas no conservadas que podrían alcanzar los $45-55 \mathrm{~cm}$ de longitud (Martínez Rubio 2006: 82-84, fig. 10). Dudoso -por incompleto- resulta el posible paralelo de Cantalar I (Bea y Domingo 2009: 41, fig. 7). En este caso se documentan dos trazos lineales, uno horizontal y de 1-1,5 cm de grosor y otro vertical, más delgado, que se une en su zona medial con el anterior. El trazo horizontal, en su zona derecha, se ve cortado por una espesa capa negra, muy posiblemente del ennegrecimiento por ahumado, que recubre la pared. Los elementos aludidos se interpretaron como el brazo y el arco de un antropomorfo en actitud de disparo.

Trab. Prehist., 70, N. ${ }^{\circ}$ 1, enero-junio 2013, pp 166-174, ISSN: 0082-5638

doi: $10.3989 /$ tp.2013.12107 

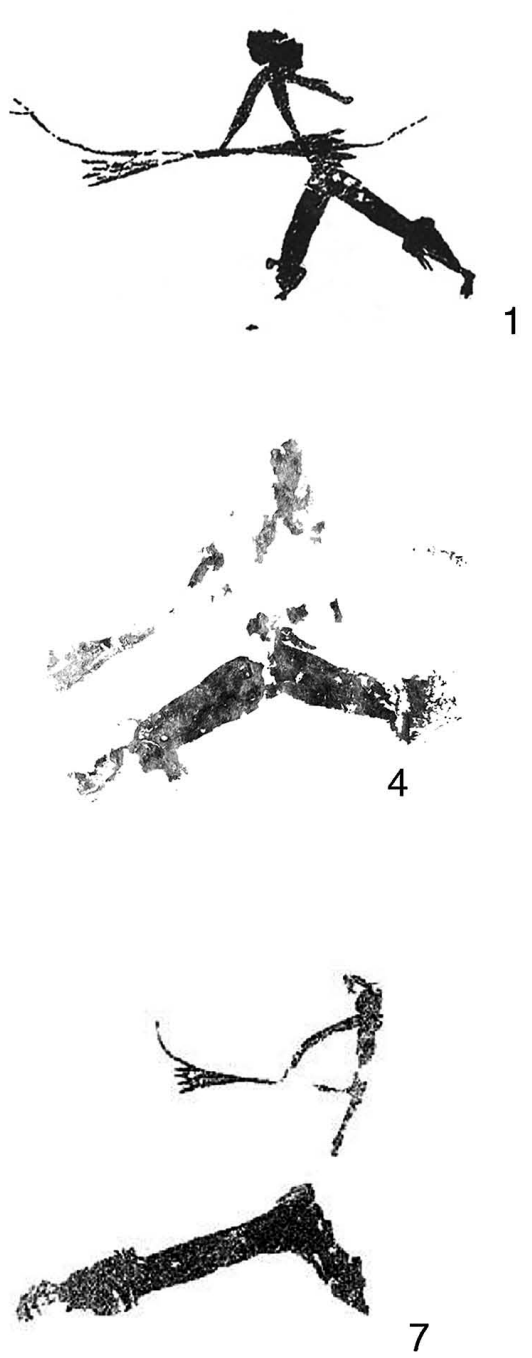
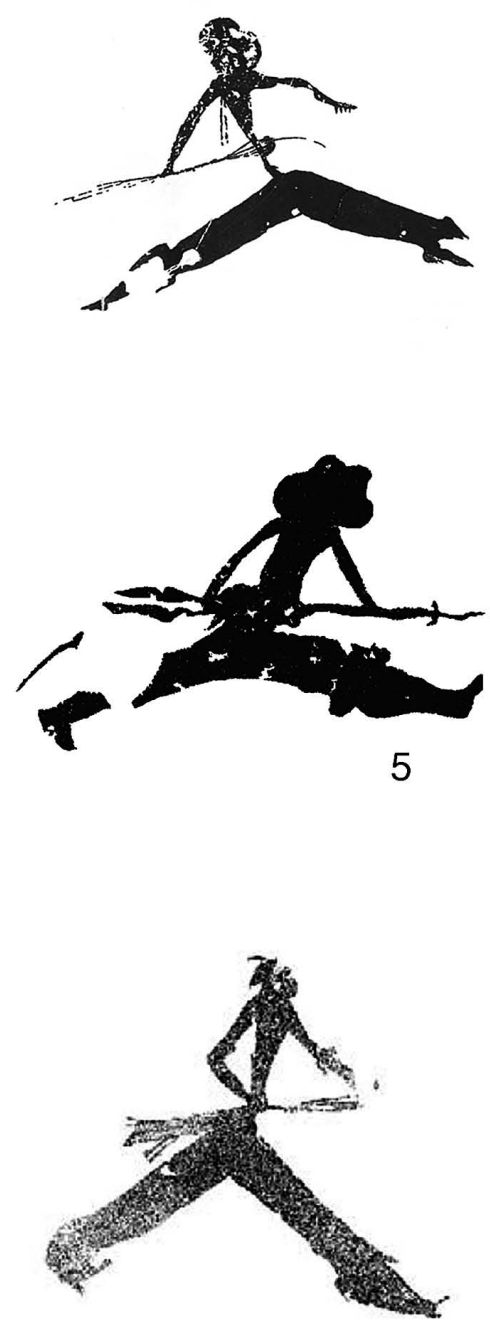
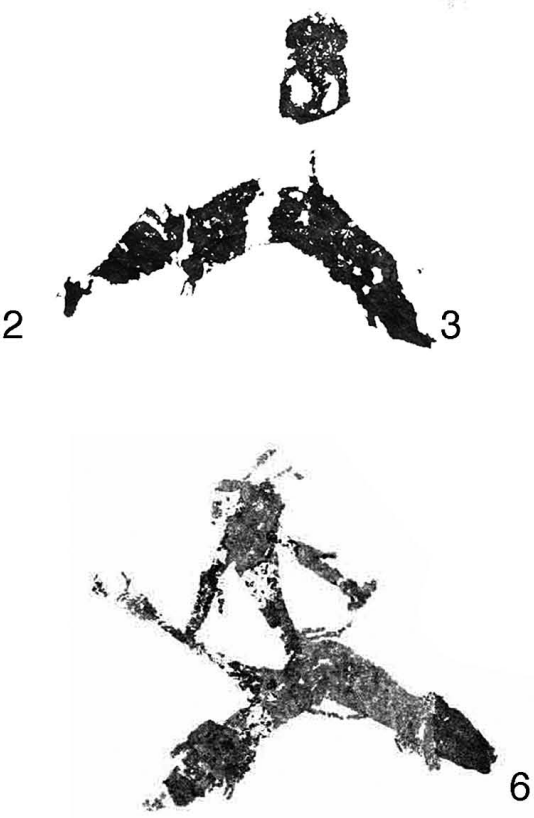

8

Fig. 3. Tratamiento del arquetipo robusto de arquero levantino o tipo Centelles: 1. y 2. Val del Charco (motivos 3 y 21, según Beltrán 2002); 3. La Vacada (según Martínez-Bea 2009); 4. Valleta del Serradó (según Bea 2012a); 5. El Garroso (según Beltrán 2005); 6. Mas del Ous (según Villaverde et al. 2006: 184, fig. 4); 7. y 8. Centelles (según Guillem y Martínez Valle 2004); 9. Els Cavalls (según Martínez Valle y Villaverde 2002).

Hasta el momento, el arquero de Val del Charco es verdaderamente un unicum en el arte levantino, con toda la problemática que los casos singulares lleva aparejada. Pero la variabilidad formal e incluso dimensional de las representaciones humanas levantinas de etapas antiguas (tipo Centelles y Civil) encuentra salvedades tan importantes como las de la Cueva del Chopo (Picazo et al. 2001-2002). Allí volvemos a encontrar representaciones humanas que superan el metro de longitud, clasificados como 'extralongilíneos' por la gran desproporción de unos cuerpos extremadamente alargados (Martínez-Bea 2005; Utrilla y Martínez-Bea 2007). El arquero que estudiamos es la representación conocida de mayor tamaño de todo el arte levantino, superando ampliamente las dimensiones calculadas para las grandes representaciones humanas mencionadas. Pero el valor simbólico de la figura transciende sus proporciones. Por su localización en el panel decorado (Fig. 4) es el elemento de mayor importancia de todo el conjunto: ocupa la posición más relevante, el cen- 
tro físico del abrigo y la zona más alta. Preside el espacio ornamental y sus dimensiones en el momento de su ejecución, debieron permitir su visibilidad a una distancia considerable, desde el otro lado del barranco, convirtiéndose así en el elemento identificador del abrigo. En este sentido, destacamos que el motivo 43 de la cavidad II de Cueva de la Araña ocupa igualmente una posición central en el panel decorado, si bien en su zona baja.

Las características apuntadas deberían ponerse en relación con la interpretación del abrigo de Val del Charco como un centro de agregación o espacio recurrente, lo que evidenciaría una organización jerárquica del territorio articulada a partir de distintas tipologías de abrigos. La incuestionable dificultad, ya apuntada (Bea 2011), que supone ensayar una jerarquización de las estaciones rupestres con el registro iconográfico conservado, sin duda incompleto, no excluye que consideremos factible una aproximación a la realidad diferenciadora de las estaciones decoradas, al menos para el territorio aragonés. Para ello nos basamos en la hipótesis de que un mayor número de representaciones levantinas en un abrigo -sobre todo si se identifican motivos propios de diferentes momentos del mismo ciclo artístico- permitiría suponer una mayor importancia simbólica del mismo, aspecto contemplado en otros estudios desde una perspectiva complementaria, atendiendo a la relación entre el número de representaciones y la ubicación de los abrigos en el paisaje (Cruz Berrocal 2005: 376-377). Con ello, adjudicamos una escala de valores a los espacios ocupados de manera recurrente a lo largo del tiempo. El mapa de intensidades resultante apunta a la existencia de una verdadera planificación de los conjuntos decorados y de su distribución en el territorio. Los 'grandes centros' o lugares de agregación, que aglutinan el mayor número de representaciones, aparecen como cabeceras de una red de estaciones rupestres satélite, desarrollada en un área más o menos amplia (Bea 2011: 288-289). Val del Charco actuaría como centro de agregación de una zona extensa, el curso bajo del río Guadalope, de especial relevancia por sus evidentes contactos temáticos y estilísticos con territorios cercanos, como el curso medio del Guadalope (alrededores de Santolea) al Sur y cuenca del Matarraña al Este (Fig. 1), sin olvidar las conexiones manifiestas con algunos de los conjuntos de Castellón y de la zona central del territorio valenciano.

Destacamos igualmente las otras representaciones de grandes dimensiones que hay en el abrigo: la 21 (arquero) con 46,5 cm de longitud, la 30 (toro) con $60 \mathrm{~cm}$, la 90 (ciervo) que alcanza los $80 \mathrm{~cm}$ de longitud en lo conservado y la 104 (mujer) con $50 \mathrm{~cm}$ de altura. Todas ellas superan ampliamente la media de las dimensiones de las representaciones temáticamente afines, aunque sin llegar a las proporciones del arquero que preside el conjunto. Las figuras citadas podrían guardar algún tipo de relación con el arquero central, ya que los motivos 21 y 30 se hallan a su izquierda y marchan en esa dirección, mientras que los motivos 90 y 104 se localizan a la derecha y se

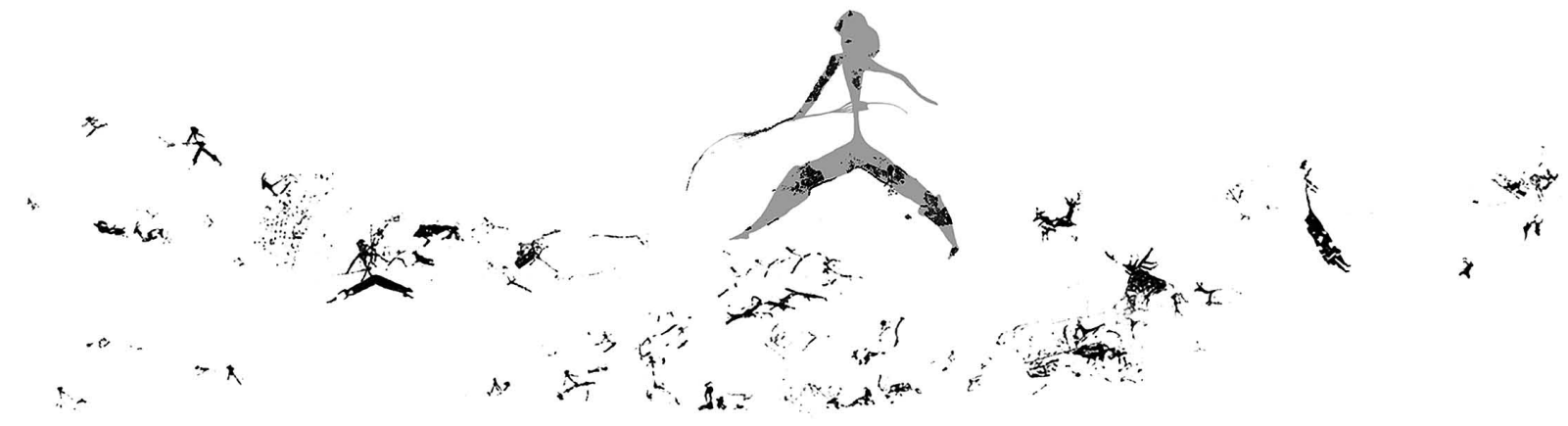

Fig. 4. Calco general del abrigo de Val del Charco en el que se indica la posición preeminente del arquero estudiado (base a partir de original de Beltrán 2002). El motivo se destaca por sus dimensiones y por ocupar el espacio central y superior del panel decorado.

Trab. Prehist., 70, N. ${ }^{\circ}$ 1, enero-junio 2013, pp 166-174, ISSN: 0082-5638 doi: $10.3989 /$ tp.2013.12107 
orientan en dicho sentido. Tanto si los motivos referidos se ejecutaron en la misma fase, como si se fueron agregando, mantienen un equilibrio o simetría con el gran arquero como eje central.

La aplicación de DStretch para ImageJ al estudio del arte levantino da lugar a interesantes resultados, permitiéndonos advertir restos pictóricos o detalles inapreciables a simple vista. Los beneficios de usar esta herramienta resultan meridianos, tanto si los motivos están muy degradados, como el analizado en este trabajo, como en un grado óptimo de conservación. El nuevo estudio del antropomorfo de Val del Charco nos ha permitido consolidar su interpretación como un verdadero arquero levantino, asignable por sus características estilísticas al arquetipo robusto u Horizonte Centelles. La circunstancia de que sea la figura humana de mayores dimensiones de todo el arte levantino determinó y complicó su propia definición con anterioridad. Sin embargo, la consideración individualizada de cada una de las partes conservadas, el análisis integrador de las mismas, así como la caracterización estilística detallada que la aproxima a representaciones humanas del propio abrigo apuntan al acierto de su interpretación como un arquero. Este motivo aparecería como el elemento dominante $\mathrm{y}$, quizá, aglutinador de todo el conjunto, así como el referente visual del entorno inmediato e incluso del territorio a una escala regional como espacio de agregación.

En ningún momento, nuestro análisis sugiere que se deba reconsiderar la definición de todo el horizonte Centelles. Como se ha apuntado, está bien expresado en estudios recientes y nunca un caso único puede ser determinante en la formulación de una generalidad. En cambio, sí pensamos que, para el momento estilístico referido, se debería destacar el recurso a singularidades temáticas (amplios grupos humanos en movimiento, sujetos ajusticiados, uso de herramientas poco comunes como el bumerán...) y estilísticas (cuerpos extralongilíneos de la Cueva del Chopo, enormes medidas globales del arquero de Val del Charco). Tales singularidades, temáticas, estilísticas o de tamaño, podrían ser un rasgo identitario e identificativo más en la definición general del horizonte Centelles y bien podrían explicarse en términos simbólicos y de significado o funcionales. En todo caso, el horizonte Centelles merece ser observado desde distintas dimensiones.

\section{AGRADECIMIENTOS}

A la Dra. Pilar Utrilla (Universidad de Zaragoza) y a los Drs. Valentín Villaverde (Universidad de Valencia) y José Luis Sanchidrián (Universidad de Córdoba) por la lectura de la versión previa del estudio y los comentarios acerca del mismo. Los comentarios y sugerencias de los evaluadores del trabajo han permitido mejorar la versión final del mismo. La investigación ha sido financiada por el proyecto HAR2001-27197 del Ministerio de Ciencia e Innovación (MICINN).

\section{BIBLIOGRAFÍA}

Almagro, M. 1956: "Las estaciones de arte rupestre del Bajo Aragón". En M. Almagro, A. Beltrán y E. Ripoll: Prehistoria del Bajo Aragón. Instituto de Estudios Turolenses. Zaragoza: 43-95.

Bea, M. 2011: "Recurrent sites and territorial hierarchy in the Levantine rock-art of Aragón". En J. J. García, H. Collado y G. Nash (eds.): The question Levantine: new directions in understanding the Spanish Levantine Rock Art. Archaeolingua. Budapest: 283-298.

Bea, M. 2012a: "Nuevas perspectivas de análisis para el arte levantino del Maestrazgo. Los abrigos del Arquero y del Torico (Castellote, Teruel, España)". Zephyrus LXX: 49-67.

Bea, M. 2012b: Arte rupestre de la Comarca del Matarraña/Matarranya. Comarca del Matarraña/Matarranya, Ministerio de Educación, Cultura y Deporte. Teruel.

Bea, M. y Domingo, R. 2009: "Las pinturas levantinas del abrigo de El Cantalar I (Villarluengo-Montoro de Mezquita, Teruel)". Saguntum 41: 37-46.

Beltrán, A. 1968: Arte Rupestre Levantino. Monografías Arqueológicas IV, Universidad de Zaragoza. Zaragoza.

Beltrán, A. 1970: La cueva del Charco del Agua Amarga y sus pinturas levantinas. Anejo de Caesaraugusta, Monografías Arqueológicas VII, Universidad de Zaragoza, Institución Fernando el Católico. Zaragoza.

Beltrán, A. 2000: "El portador de animal núm. 55 del abrigo 'levantino' de Val del Charco: rectificaciones sobre una figura humana mal publicada". Cuadernos de Prehistoria y Arqueología Castellonenses 21: 69-75.

Beltrán, A. 2002: Las pinturas rupestres del abrigo de Val del Charco del Agua Amarga de Alcañiz. Prames. Zaragoza.

Beltrán, A. 2005: Corpus de arte rupestre del Parque Cultural del río Martín. Parque Cultural del río Martín. Zaragoza.

Cabré, J. 1915: Arte rupestre en España. Memoria 1 Comisión de Investigaciones Paleontológicas y 
Prehistóricas, Junta para Ampliación de Estudios e Investigaciones Científicas, Instituto Nacional de Ciencias Físico-Naturales, Museo Nacional de Ciencias Naturales. Madrid.

Cruz Berrocal, M. 2005: Paisaje y arte rupestre: ensayo de contextualización arqueológica y geográfica de la pintura levantina. Universidad Complutense de Madrid, Servicio de Publicaciones. Madrid. http://www.ucm.es/BUCM/tesis/ghi/ucm-t27480. pdf (consulta 10-II-2012).

Díaz-Andreu, M. 2012: "Recording biographies. New research into two schematic rock art sites in Murcia (Spain)". International Newsletter on Rock ART (INORA) 62: 20-27.

Domingo, I. 2005: Técnica de ejecución de la figura en el arte rupestre levantino. Hacia una definición actualizada del concepto de estilo: validez y limitaciones. Universitat de València. València. http:// tdx.cat/handle/10803/10173 (consulta 10-II-2012).

Domingo, I. 2006: "La figura humana, paradigma de continuidad y cambio en el arte rupestre levantino". Archivo de Prehistoria Levantina XXVI: 161-191.

Domingo, I. y López, E. 2002: "Metodología: el proceso de obtención de calcos o reproducciones". En R. Martínez Valle y V. Villaverde (eds.): La Cova dels Cavalls en el Barranc de la Valltorta. Monografías del Instituto de Arte Rupestre 1, Museu de la Valltorta. Tírig: 75-81.

Guillem, P. y Martínez-Valle, R. 2004: "Las figuras humanas del abrigo del Barranco Hondo en el contexto del arte levantino del Bajo Aragón-Maestrazgo". En P. Utrilla y V. Villaverde (eds.): Los grabados levantinos del Barranco Hondo (Castellote, Teruel), Monografías del Patrimonio Aragonés 1, Gobierno de Aragón, Zaragoza: 105-122.

Harman, J. 2005: "Using decorrelation Stretch to enhance rock art images". American Rock Art Research Association Annual Meeting (May 28, 2005). Reno. http://www.petroglyphs.us/article_ using_decorrelation_stretch_to_enhance_rock_ art_images.htm (consulta 10-II-2012).
Hernández-Pacheco, E. 1924: Las pinturas prehistóricas de las Cuevas de La Araña (Valencia). Memoria 34, Comisión de Investigaciones Paleontológicas y Prehistóricas, Junta para Ampliación de Estudios e Investigaciones Científicas, Museo Nacional de Ciencias Naturales. Madrid.

Martínez-Bea, M. 2005: Variabilidad estilística y distribución territorial del arte rupestre levantino en Aragón: el caso de La Vacada (Castellote, Teruel). Universidad de Zaragoza. Zaragoza.

Martínez-Bea, M. 2009: Las pinturas rupestres del abrigo de La Vacada (Castellote, Teruel). Monografías Arqueológicas 43, Universidad de Zaragoza. Zaragoza.

Martínez-Rubio, T. 2006: "El Cinto de las Letras (Dos Aguas), revisió del panell 55 anys després de la seua publicació". Saguntum 38: 61-91.

Martínez Valle, R. y Villaverde, V. 2002: La Cova dels Cavalls en el Barranc de la Valltorta. Monografías del Instituto de Arte Rupestre 1. Museu de la Valltorta. Tírig.

Picazo, J. V.; Loscos, R. M.; Martínez-Bea, M. y Perales, P. 2001-2002: "Las pinturas rupestres de la Cueva del Chopo". Kalathos 20-21: 27-83.

Royo, J. I. y Benavente, J. A. 1999: Val del Charco del Agua Amarga. Un modelo para la protección y difusión del arte rupestre aragonés. Ayto. de Alcañiz y Gobierno de Aragón. Zaragoza.

Tomás Maigí, J. 1951: "Del Charco del Agua Amarga (Alcañiz)". Zephyrus II: 5-13.

Utrilla, P. 2005: "Arte rupestre en Aragón. 100 años después de Calapatá". En M. S. Hernández y J. A. Soler (eds.): Arte rupestre en la España mediterránea (Alicante 2004): 341-377. Alicante.

Utrilla, P. y Martínez-Bea, M. 2007: "La figura humana en el arte rupestre aragonés". Cuadernos de Arte Rupestre 4: 161-203.

Villaverde, V.; Guillem, P. y Martínez Valle, R. 2006: "El horizonte gráfico Centelles y su posición en la secuencia del Arte Levantino del Maestrazgo". Zephyrus LIX: 181-198. 